\section{Evaluating endophenotypes for psychiatric disorders}

\author{
Avaliação de \\ endofenótipos em \\ transtornos psiquiátricos
}

Studies on the etiology and pathophysiology of psychiatric disorders and studies on factors associated with treatment response have both been limited by clinical and genetic heterogeneity. The validity of diagnostic criteria based on clinical symptoms has been difficult to achieve because the psychiatric disorders, as defined by current classification schemas including DSM, likely encompass a group of disorders that are heterogeneous with respect to etiology and pathophysiology. In fact, without knowledge of the underlying etiological processes the diagnostic criteria for a disorder based on clinical characteristics cannot be evaluated adequately. The use of more indirect indicators of disease validity including family history, treatment response, longitudinal course and stability, patterns of comorbidity, and social consequences led to circularity in validating clinical diagnostic criteria. ${ }^{1}$ The evaluation of endophenotypes (i.e., intermediate phenotypes bridging the gap between available descriptors and the elusive underlying etiology) has been proposed as a strategy to overcome the methodological difficulties in psychiatric nosology and has already been a successful strategy in the elucidation of genetic factors in schizophrenia and medical diseases. ${ }^{2}$

The multifactorial threshold model of complex genetic disease assumes that many factors contribute to a disorder in an individual, that the effects of each single factor may be small but small effects may accumulate, and that once the combined effects of the factors pass some critical value the disorder may become manifest. ${ }^{3}$ This model also can be applied to both macro phenotypes and endophenotypes since we assume that multiple genetic and non-genetic factors contribute to a complex phenotype that becomes manifest when the combined effects of the factors pass the phenotype-specific threshold. The endophenotype approach assumes that the underlying liability of endophenotypes representing basic biological phenomena is less complex and easier to elucidate than the liability of complex behaviors, such as psychiatric diseases, irrespective of the magnitude of the total genetic risk of the phenotypic definition. Genetic factors for intermediate traits that are closer to the genotype in the developmental scheme may generally be easier to identify because of the improved signal-to-noise ratio in the fraction of variance explained by any single factor.

Although the endophenotype concept has been specifically developed to improve the power of genetic studies, the identification of endophenotypes will also lead to improvements of diagnostic methods, disease classification, the development of animal models, and the development of better treatments. For example, assuming that neurologists would not know the different etiologies and pathophysiologies of tremor, they might define a 'major tremor disorder' based on clinical characteristics. As a result, they would include patients with conditions as dissimilar as classic Parkinson's disease, multiple system atrophy, progressive supranuclear palsy, and frontotemporal dementia in the same trial testing the effect of a dopamine agonist in major tremor disorder. Although clinicians may recognize that some patients (i.e., those with Parkinson's disease) experience considerable symptom reductions under treatment, the treatment effect across the whole heterogeneous sample would not reach statistical significance, and the effective drug would not be allowed 
for use in Parkinson's disease. Sadly, this example may reflect the current situation in psychiatry.

There is no reliable method to select behavioral or biological markers for studies aimed at evaluating endophenotypes. Generally, a marker should be selected with respect to 1) feasibility and reliability of its measurement; 2) high heritability; 3) availability of knowledge on its underlying neurobiology/genetics; and 4) possible relevance for the disorder under study. Criteria to evaluate endophenotypes were developed by Gottesman and associates. The most recent version of the criteria is published in. ${ }^{4}$

1) An endophenotype is associated with illness in the population. Usually, this criterion is examined by case-control studies, although community-based studies are clearly preferable because they provide more reliable and representative estimates of association. Since endophenotypes reflect genetically relevant aspects of the heterogeneous pathophysiology of the disease, they are different from diagnostic markers, which are evaluated by measures of sensitivity and specificity. In psychiatry, endophenotypes are clearly different from diagnostic markers because it cannot be assumed that the current definitions of psychiatric diseases are genetically valid.

2) An endophenotype is heritable. Twin studies provide estimates of the relative importance of genetic factors in the development of a marker. Frequently, researchers rely on heritability of general constructs such as general intellectual functioning because the heritability of the specific cognitive function under study is not available. It must be kept in mind, however, that the heritability of a specific brain function might be considerably lower than that of a more general brain function.

3) An endophenotype is state-independent. Longitudinal studies test this criterion. Given the increasing recognition of phenotypic changes over time, ${ }^{5}$ the criterion 'state-independence' might be particularly difficult to achieve for candidate endophenotypes. Age-normed definitions of endophenotypes and symptom provocation methods (e.g. glucose tolerance test in diabetes and tryptophan depletion in depression) may be necessary to fulfill this criterion. ${ }^{5}$

4) Within families, endophenotype and illness co-segregate. Relatively large family studies may be needed to test this criterion in genetically complex diseases.

5) An endophenotype identified in probands is found in their unaffected relatives at a higher rate than in the general population. The high-risk design, which compares prevalence rates of a marker between subjects at familial risk of the disease and those without the risk, is a powerful strategy to address this criterion.

Given the lack of well-designed twin, family, and prospective studies evaluating putative endophenotypes in psychiatric disorders, future research has the potential to considerably improve the phenotypic definition of these highly prevalent conditions that frequently have a devastating effect on quality of life and life expectancy.

Gregor Hasler Department of Psychiatry, University Hospital, Culmannstrasse, Zurich, Switzerland

\section{References}

1. Hasler G, Drevets WC, Manji HK, Charney DS. Discovering endophenotypes for major depression. Neuropsychopharmacology. 2004;29(10):1765-81.

2. Gottesman II, Gould TD. The endophenotype concept in psychiatry: etymology and strategic intentions. Am J Psychiatry. 2003;160(4):636-45.

3. Gottesman II, Shields J. A polygenic theory of schizophrenia. Proc Natl Acad Sci U S A. 1967,58(1):199-205.

4. Hasler G, Drevets WC, Gould TD, Gottesman, II, Manji HK. Toward Constructing an Endophenotype Strategy for Bipolar Disorders. Biol Psychiatry. 2006; [Epub ahead of printing].

5. Hasler G, Pine DS, Kleinbaum DG, Gamma A, Luckenbaugh D, Ajdacic V, Eich D, Rossler W, Angst J. Depressive symptoms during childhood and adult obesity: the Zurich Cohort Study. Mol Psychiatry. 2005; 10(9):842-50. 